\title{
ИЗУЧЕНИЕ АКТИВНОСТИ КАТАЛИТИЧЕСКИХ СИСТЕМ НА ОСНОВЕ БИСЦИКЛОПЕНТАДИЕНИЛЬНЫХ СОЕДИНЕНИИ ТИТАНА ПРИ ПОЛИМЕРИЗАЦИИ ЭТИЛЕНА *
}

\author{
X. KAAP \\ О. КИРРЕТ, \\ член-корреспондент Академии наук Эстонской ССР \\ Г. ШВИНДЛЕРМАН
}

\section{2. Нзученне активности каталитического комплекса}

$$
\left(\mathrm{C}_{5} \mathrm{H}_{5}\right)_{2} \mathrm{TiCl}_{2}-\left(\text { uзo- } \mathrm{C}_{4} \mathrm{H}_{9}\right)_{2} \mathrm{AlCl}
$$

Прн полимеризации этилена на свежеприготовленном комплексе $\left(\mathrm{C}_{5} \mathrm{H}_{5}\right)_{2} \mathrm{TiCl}_{2}-$ (изо- $\left.\mathrm{C}_{4} \mathrm{H}_{9}\right)_{2} \mathrm{AlCl}$ при атмосферном давлении в инертном растворителе получают полиэтилен со строго линейным строением (содержит $0,05 \%$ метильных групп) и высокой температурой плавления $\left(137^{\circ}\right)$. Однако активность комплекса по сравнению с гетерогенными катализаторами типа $\mathrm{R}_{3} \mathrm{Al}$ или $\mathrm{R}_{2} \mathrm{AlCl}+\mathrm{TiCl}_{4}$ или $\mathrm{TiCl}_{3}$ низка. В литературе описано несколько способов повышения активности этой системы - введение в реакционную смесь следов кислорода [1-3], $\mathrm{HCl}$ и воды [4, 5], но причины увеличения скорости полимеризации в присутствии добавок не выяснены.

В данной работе будут рассмотрены возможности увеличения активности каталнтического комплекса. $\left(\mathrm{C}_{5} \mathrm{H}_{5}\right)_{2} \mathrm{TiCl}_{2}-\left(\text { изо } \cdot \mathrm{C}_{4} \mathrm{H}_{9}\right)_{2} \mathrm{AlCl}$, связанные с действием добавки $\mathrm{HCl}$.

\section{Экспериментальная часть}

Подготовку реактивов и катализаторов, а также полимеризацию этилена в проточной системе проводили по методике, описанной в [']. (изо- $\left.\mathrm{C}_{4} \mathrm{H}_{9}\right){ }_{2} \mathrm{AlCl}$ и изо $-\mathrm{C}_{4} \mathrm{H}_{9} \mathrm{AlCl}_{2}$ синтезировали в атмосфере аргона из очищенного, перегнанного в вакууме (изо- $\left.\mathrm{C}_{4} \mathrm{H}_{9}\right)_{3} \mathrm{Al}$ I свежевозогнанного $\mathrm{AlCl}_{3}$ (избыток $5 \%$ против стехиометрического количества), в среде $\boldsymbol{H}$-гептана при $\sim 50^{\circ}$. (изо- $\left.\mathrm{C}_{4} \mathrm{H}_{9}\right)_{2} \mathrm{AlCl}$ очищали перегонкой в вакууме (температура кнпення $\left(\text { изо- } \mathrm{C}_{4} \mathrm{H}_{9}\right)_{2} \mathrm{AlCl}-116-118^{\circ} / 2-3$ мм); изо- $\mathrm{C}_{4} \mathrm{H}_{9} \mathrm{AlCl}_{2}$ перекристаллизовали из гептана. Қачество продуктов и концентрацию рабочих растворов проверяли аналитически по соотношению $\mathrm{R}: \mathrm{Al}: \mathrm{Cl}$, разлагая пробу $20 \% \mathrm{HNO}_{3}$ с последующим измерением объема выделившегося изобутана, трилонометрическим определением $\mathrm{Al}\left[{ }^{6}\right]$ и определением $\mathrm{Cl}$ по фольгарду.

Активность каталитической системы оценивалась по кинетическим кривым и по выходу полимера в граммах на 1 моль $\left(\mathrm{C}_{5} \mathrm{H}_{5}\right)_{2} \mathrm{TiCl}_{2}$ за время опыта (1,5 часа).

* Продолжение сообщения, опубликованного в Изв. АН ЭССР. Сер. физ.матем. и техн. наук, 1963, 3 .

Примеч ан ие: В статье $\mathrm{O}$ каталитической активности системы $\mathrm{R}_{3} \mathrm{Al}-$ $\left(\mathrm{C}_{5} \mathrm{H}_{5}\right)_{2} \mathrm{TiCl}_{2}$ при полимеризации этилена». Изв. АН ЭССР. Сер. физ.-матем. и техн. наук, 1963, № 3, на стр. 296 в табл. 1 в названни графы «Выход полимера г/лоль $\left(\mathrm{C}_{5} \mathrm{H}_{5}\right) \mathrm{TiCl}_{2}$ » следует читать «Выход полимера г/0,1 моля $\left(\mathrm{C}_{5} \mathrm{H}_{5}\right)_{2} \mathrm{TiCl}_{2}$ ». 


\section{Обсуждение результатов}

При взанмоден̆ствни $\mathrm{R} 2 \mathrm{AlCl}$ с $\left(\mathrm{C}_{5} \mathrm{H}_{5}\right)_{2} \mathrm{TiCl}_{2}$, как известно, пронсходит последовательног образованне ряда лабильных комплексов, сопровождающееся измененнем окраски, прнводящее в конечном итоге к образованию неактивного (или малоактивного) зеленого комплекса, в котором $\mathrm{Ti}$ восстановлен до трехвалентного состояния и отсутствуют связи $\mathrm{C}-\mathrm{Ti}$, по которым ндет рост полимерных мепей. Каталитическая актнвность системы связана с одним из промежуточных комплексов. B ряду $\mathrm{R}_{3} \mathrm{Al}, \mathrm{R}_{2} \mathrm{AlCl}$, $\mathrm{RAlCl}_{2}$ наиболее актнвный каталнзатор с $\left(\mathrm{C}_{5} \mathrm{H}_{5}\right)_{2} \mathrm{TiCl}_{2}$ дает $\mathrm{R}_{2} \mathrm{AlCl}$, поскольку с участием $\mathrm{R}_{2} \mathrm{AlCl}$ образование активных структур пронсходит достаточно быстро, а их распадд сравннтельно медленно. С (изо- $\left.\mathrm{C}_{4} \mathrm{H}_{9}\right)_{2} \mathrm{AlCl}$ при $20^{\circ}$ в среде толуола конечный комплекс образуется за 60 мин, тогда как в таких же условиях с участием (изо$\left.\mathrm{C}_{4} \mathrm{H}_{9}\right)_{3} \mathrm{Al}$ он образуется за 2 мин.

- Так как концентрация активных центров в реакционной смеси низ̆ка $\left[{ }^{7}, 8\right]$, то скорость полимеризацни на комплексе (uso- $\left.\mathrm{C}_{4} \mathrm{H}_{9}\right)_{2} \mathrm{AlCl}-\left(\mathrm{C}_{5} \mathrm{H}_{5}\right)_{2} \mathrm{TiCl}_{2}$ остается малой. :Нанбольшую активность снстема нмеет в интервале температур $+20^{\circ}-$ $+40^{\circ}$ (рис. 1 ).

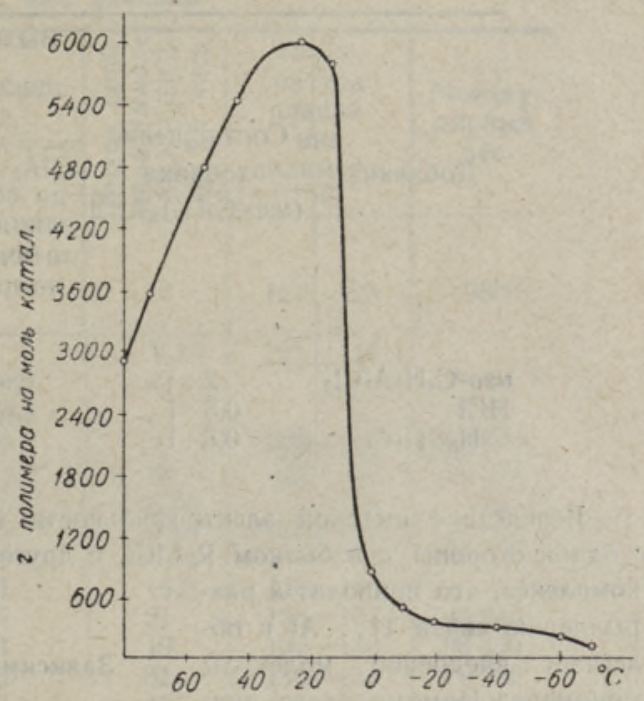

Рис. 1. Зависимость выхода полимера от температуры:

$\left(\mathrm{C}_{5} \mathrm{H}_{5}\right)_{2} \mathrm{TiCl}_{2}+2$ ммоля/ $\Omega$;

(изо- $\left.\mathrm{C}_{4} \mathrm{H}_{9}\right)_{2} \mathrm{AlCl}-8$ ммолей $/ \Omega$.

Растворитель - толуол. Время 7 часов.

Повышение температуры до $+70^{\circ}$ или выше не увеличивает, активности катализатоpa, а лишь ускоряет распад связей $\mathrm{C}-\mathrm{Ti}$ и образование малоактивного конечного комплекса. Понижение температуры ниже $0^{\circ}$ настолько уменьшает скорость формировання активных центров, что катализатор практически теряет активность. Известно, что $\mathrm{HCl}$ (используемый в качестве активирующей добавки к данному комплексу) не действует на $\left(\mathrm{C}_{5} \mathrm{H}_{5}\right)_{2} \mathrm{TiCl}_{2}$, однако в алкилалюминиевых соединениях при действии $\mathrm{HCl}$ пронсходит вытеснение алкильных групп хлором. Поэтому казалось вероятным, что активированне каталитической системы $\left(\mathrm{C}_{5} \mathrm{H}_{5}\right)_{2} \mathrm{TiCl}_{2}-\mathrm{R}_{2} \mathrm{AlCl}$ небольшими количествами $\mathrm{HCl}$ связано с превращением части $\mathrm{R}_{2} \mathrm{AlCl}$ в $\mathrm{RAlCl}_{2}$. Так как между $\mathrm{RAICl}_{2}$ и $\mathrm{R}_{2} \mathrm{AlCl}$ пронсходит быстрый обмен радикалами и образуются смешанные димеры [ $\left.{ }^{9}\right]$, то можно предполагать, что добавка $\mathrm{RAlCl}_{2}$ способствует дальнейшему снижению восстановительной способности $\mathrm{R}_{2} \mathrm{AlCl}$ и, таким образом, увеличивает время жизни активных центров. Однако, если ввести в реакционную смесь как $\mathrm{HCl}$, так и $\mathrm{RAlCl}_{2}$ до образования каталитического комплекса, то полимеризация этилена не проходит, хотя зеленый комплекс образуется. Если предварительно смешать алюминийалкил с $\mathrm{HCl}$ или $\mathrm{RAICl}_{2}$, то полимернзация идет, но скорость поглощения мономера низка. Заметное увеличение активности катализатора имеет место лишь в том случае, если $\mathrm{HCl}$ или $\mathrm{RAlCl}_{2}$ добавляют к готовому комплексу $\left(\mathrm{C}_{5} \mathrm{H}_{5}\right)_{2} \mathrm{TiCl}_{2}-\mathrm{R}_{2} \mathrm{AlCl}$ сразу же после его формировання (габл. 1, 2). На основе этого можно сделать вывод, что активирующее действие связано с электрофильной атакой готового каталитического комплекса, в котором уже есть связь С-Ті. Қаталитически активной является, скорее всего, равновесная (между мостиковой и нонной структурой) форма комплекса

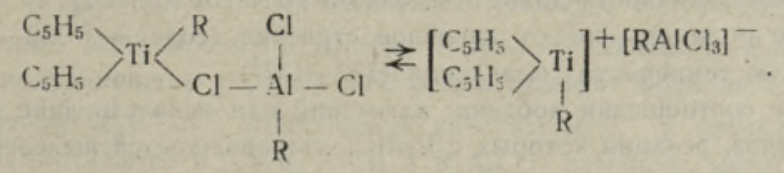


Зависимость выхода полимера от последовательности введения добавки

\begin{tabular}{|c|c|c|c|c|}
\hline \multirow[b]{3}{*}{ Добавка } & \multirow{3}{*}{$\begin{array}{l}\text { Соотношение } \\
\frac{\text { добавка }}{\left(\text { изо- } \mathrm{C}_{4} \mathrm{H}_{9}\right)_{2} \mathrm{AlCl}}\end{array}$} & \multirow{2}{*}{\multicolumn{3}{|c|}{$\begin{array}{c}\text { Выход полимера г/мноль }\left(\mathrm{C}_{5} \mathrm{H}_{5}\right)_{2} \mathrm{TiCl}_{2} \\
\text { за } 1,5 \text { часа } \\
\text { В веден и е до ба в ки }\end{array}$}} \\
\hline & & & & \\
\hline & & $\begin{array}{l}\text { до образо- } \\
\text { вания ката- } \\
\text { литического } \\
\text { комплекса }\end{array}$ & $\begin{array}{l}\text { вместе с } \\
\text { (uзo- } \\
\left.\mathrm{C}_{4} \mathrm{H}_{9}\right)_{2} \mathrm{AlCl}\end{array}$ & $\begin{array}{c}\text { после обра- } \\
\text { зования ка- } \\
\text { талитиче- } \\
\text { ского комп- } \\
\text { лекса }\end{array}$ \\
\hline $\begin{array}{l}u 30-\mathrm{C}_{4} \mathrm{H}_{9} \mathrm{AlCl} \mathrm{Cl}_{2} \\
\mathrm{HCl} \\
\mathrm{C}_{6} \mathrm{H}_{5} \mathrm{CH}_{2} \mathrm{Cl}\end{array}$ & $\begin{array}{r}2: 1 \\
0,5: 1 \\
0,5: 1\end{array}$ & $\begin{array}{l}\text { следы } \\
\text { следы } \\
\text { нет }\end{array}$ & $\begin{array}{c}12 \\
5 \\
\text { следы }\end{array}$ & $\begin{array}{l}21 \\
19 \\
21\end{array}$ \\
\hline
\end{tabular}

Вследствие высокой электрофильности $\mathrm{RAlCl}_{2}$ имеет место взаимодействие есо, с одной стороны, с избытқом $\mathrm{R}_{2} \mathrm{AlCl}$, с другой стороны, с аннонной частью полярного комплекса, что приводит к разрыхлению связи $\mathrm{Ti} \ldots \mathrm{Al}$ и облегчает внедрение молекулы мономера. Помимо этого снижается алкилирующая способность аниона алюминия (в снстеме присутствуют или димеры, или соединения с одной связью R-Al), т. е. уменьшается возможность дальнейшего внутрикомплексного диалкилирования титана, приводящего к быстрому распаду связи $\mathrm{Ti}-\mathrm{C}$. и восстановлению титана. Если смешать $\mathrm{R}_{2} \mathrm{AlCl}$ с $\mathrm{RAlCl}_{2}$ до образования комплекса $\mathrm{R}_{2} \mathrm{AlCl}$ c $\left(\mathrm{C}_{5} \mathrm{H}_{5}\right)_{2} \mathrm{TiCl}_{2}$, то образуются сравнительно стабильные сме-

Таблица 2

Завнсимость выхода полимера от соотношения $\left(\text { uзo- } \mathrm{C}_{4} \mathrm{H}_{9}\right)_{2} \mathrm{AlCl}:$ uзo- $\mathrm{C}_{4} \mathrm{H}_{9} \mathrm{AlCl}_{2}$

\begin{tabular}{|c|c|c|c|}
\hline $\begin{array}{c}\text { Алкилалюминиевый } \\
\text { компонент ка- } \\
\text { талнзатора }\end{array}$ & 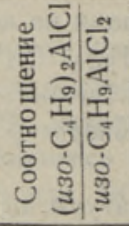 & $\begin{array}{l}\text { Раство- } \\
\text { ритель }\end{array}$ & $\begin{array}{c}\text { Выход } \\
\text { полимера } \\
\text { г/ мноль } \\
\left(\mathrm{C}_{5} \mathrm{H}_{5}\right)_{2} \mathrm{TiCl}_{2} \\
\text { за } 1,5 \text { ча- } \\
\text { са }\end{array}$ \\
\hline 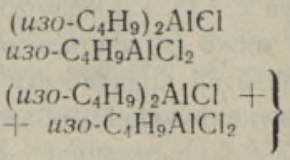 & $\begin{aligned} 2 & : 1 \\
1 & : 1 \\
0,5 & : 1\end{aligned}$ & $\begin{array}{c}\text { Толуол } \\
, " \\
", \\
", \\
,\end{array}$ & $\begin{array}{c}10 \\
\text { следи } \\
21 \\
19,5 \\
8\end{array}$ \\
\hline
\end{tabular}

Концентрация $\left(\mathrm{C}_{5} \mathrm{H}_{5}\right)_{2} \mathrm{TiCl}_{2}-2$ ммоля/л, темперагура полимеризацин - $40^{\circ}$, соотношение $\mathrm{Ti}: \mathrm{Al}-$ $1: 4$. шанные димеры, вследствие этого скорость формирования активного комплекса падает.

Поскольку известно, что многие алкилгалогениды взанмодействуют с алюмнннйорганическими соединениями, обменивая $\mathrm{R}$ на $\mathrm{Cl}$ при $\mathrm{Al}$, аналогично действию $\mathrm{HCl}$, то повышенңе активности каталитического комплекса $\left(\mathrm{C}_{5} \mathrm{H}_{5}\right)_{2} \mathrm{TiCl}_{2}-\mathrm{AlR}_{2} \mathrm{Cl}$ может быть достигнуто с помощью введения в реакционную смесь соединений типа $\mathrm{R}^{1} \mathrm{Cl}$. В этом случае активирующее действие также проявляется обычно при введении добавки немедленно вслед за смешением $\left(\mathrm{C}_{5} \mathrm{H}_{5}\right)_{2} \mathrm{TiCl}_{2}$ и $\mathrm{AlR}_{2} \mathrm{Cl}$. Результаты, полученные при использовании ряда алкнлгалогенидов в качестве активирующих добавок, приведены в табл. 3.

Увеличение скорости полимеризацин при введении в систему небольших количеств (меньше 0,5-1 ммоля на 1 ммоль $\mathrm{R}_{2} \mathrm{AlCl}$ ) $\mathrm{HCl}$ или алкилхлорндов, при взанмодействии которых с $\mathrm{R}_{2} \mathrm{AlCl}$ свободный $\mathrm{HCl}$ не выделяется, по-видимому, прежде всего обусловлено образованием $\mathrm{RAlCl}_{2}$. Полимеризация проходит с постоянной скоростью. Қатализатер не теряет активности даже в течение 5-6 часов (рис. 2).

Полученные полнмеры имеют линейное строенне (содержат $0,2-0,5 \%$ метильнғ групп) и высокую температуру плавления (133-137 $)$.

Возрастание соотношения добавка: алюминий или использование в качестве добавок алкилхлоридов, реакция которых с $\mathrm{R}_{2} \mathrm{AlCl}$ сопровождается выделеннем $\mathrm{HCl}$, в слу- 
Таблица 3

\section{Зависнмость выхода полимера на катализаторе $\left(\mathrm{C}_{5} \mathrm{H}_{5}\right)_{2} \mathrm{TiCl}_{2}-\left(\text { изо- } \mathrm{C}_{4} \mathrm{H}_{9}\right)_{2} \mathrm{AlCl}$ от характера растворителя или добавки}

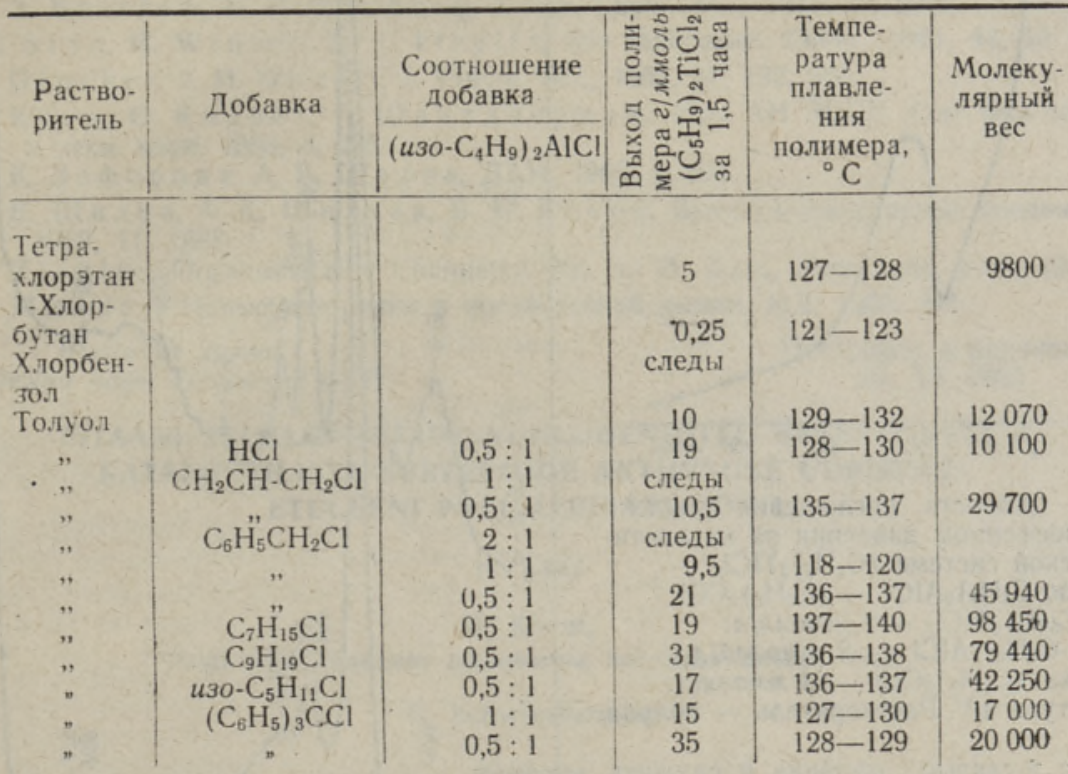

$\left(\mathrm{C}_{5} \mathrm{H}_{5}\right){ }_{2} \mathrm{TiCl}_{2}-2$ ммоля/л, (изо- $\left.\mathrm{C}_{4} \mathrm{H}_{9}\right)_{2} \mathrm{AlCl}-8$ ммолей/ $/$, объем растворителя - $100 \mathrm{M} \Omega$, температура $40^{\circ}$.

чае наличия в реакционной смеси ароматических углеводородов приводит к осложнению процесса реакциями Фриделя-Крафтса. Сильное увеличение скорости поглоцения этилена в этнх случаях объясняется скорее реакцией алкилирования растворителя мономером, нежели полимеризацией.

Рис. 2. Скорость поглощения этилена при атмосферном давлении на каталитической системе $\left(\mathrm{C}_{5} \mathrm{H}_{5}\right)_{2} \mathrm{TiCl}_{2}-$ (изо $\left.-\mathrm{C}_{4} \mathrm{H}_{9}\right)_{2} \mathrm{AlCl}-\mathrm{C}_{7} \mathrm{H}_{15} \mathrm{Cl}$ $\left(\mathrm{C}_{5} \mathrm{H}_{5}\right)_{2} \mathrm{~T}_{1} \mathrm{Cl}_{2}-2$ ммоля/л; $\left(\text { изо- } \mathrm{C}_{4} \mathrm{H}_{9}\right)_{2} \mathrm{AlCl}-8$ ммолей/л. Растворитель - толуол. Добавка $\mathrm{C}_{7} \mathrm{H}_{15} \mathrm{Cl}-4$ ммоля/л. Температура

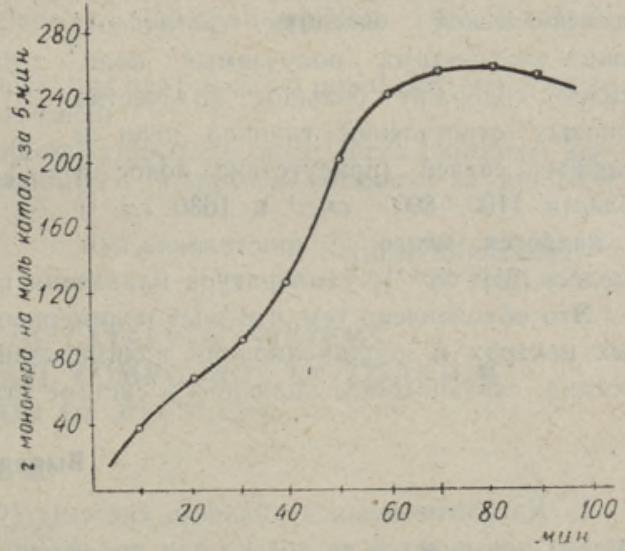
$40^{\circ}$.

Образующиеся полимеры имеют неоднородное строение, невысокий молекулярный вес и сравнительно низкую температуру плавления. Из продуктов реакции выделены алкилтолуолы.

Прибавление к каталитическому комплексу третичных алкилгалогенидов очень сильно увеличивает скорость поглощения мономера; на кинетической кривой появля ются два максимума (рис. 3). Второй максимум примерно соответствует тому времени, когда при таких же условиях в системе толуол + этилен $+\mathrm{R}_{2} \mathrm{AlCl}+\mathrm{R}_{3}^{\prime} \mathrm{CCl}$ после некоторого индукционного периода-начинается реакция Фриделя-Крафтса. Образующиеся при распаде $\mathrm{R}_{3}^{1} \mathrm{C}^{+} \mathrm{Cl}^{-}$карбониевые катионы $\mathrm{R}_{3}^{1} \mathrm{C}^{+}$в присутствии ионных комплексов (Фриделя-Крафтса и $\left(\mathrm{C}_{5} \mathrm{H}_{5}\right)_{2} \mathrm{TiCl}_{2}$ с $\left.\mathrm{R}_{2} \mathrm{AlCl}\right)$, очевидно, могут взаимодейст- 


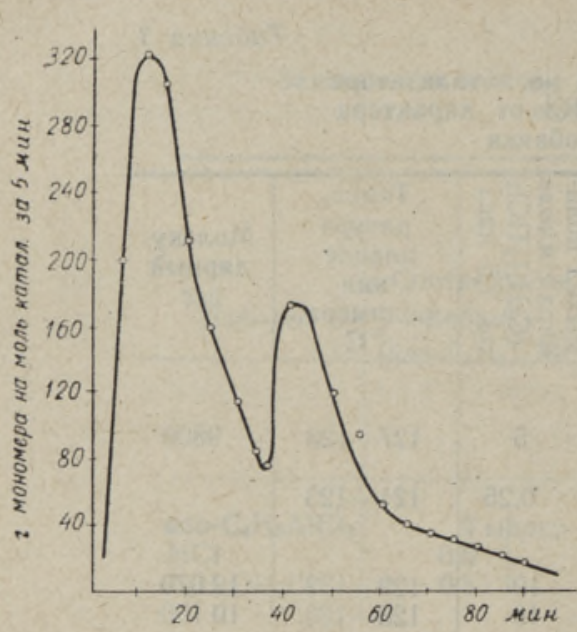

Рис. 3. Скорость поглощения этилена при атмосферном давлении на каталитической системе $\left(\mathrm{C}_{5} \mathrm{H}_{5}\right)_{2} \mathrm{TiCl}_{2}$ (uзo- $\left.\mathrm{C}_{4} \mathrm{H}_{9}\right)_{2} \mathrm{AlCl}-\left(\mathrm{C}_{6} \mathrm{H}_{5}\right)_{3} \mathrm{CCl}$. $\left(\mathrm{C}_{5} \mathrm{H}_{5}\right)_{2} \mathrm{TiCl}_{2}-2$ ммоля/л;

(изо- $\left.\mathrm{C}_{4} \mathrm{H}_{9}\right)_{2} \mathrm{AlCl}-8$ млолей/л. Добавка $\left(\mathrm{C}_{6} \mathrm{H}_{5}\right)_{3} \mathrm{CCl}-4$ мноля/л. Температура $40^{\circ}$. Растворитель - толуол. вовать с л-связью олефина и служить переносчиками цепи (10).

Инфракрасные спектры (рис. 5) показывают, что в случае промотирования каталитической системы третичными алкилгалогенидами получаемый полиэтилен содержит большое количество боковых ответвлений главной цепи и двойных связей (присутствие полос в области $1100-800 \mathrm{~cm}^{-1}$ и $\left.1630 \mathrm{~cm}^{-1}\right)$

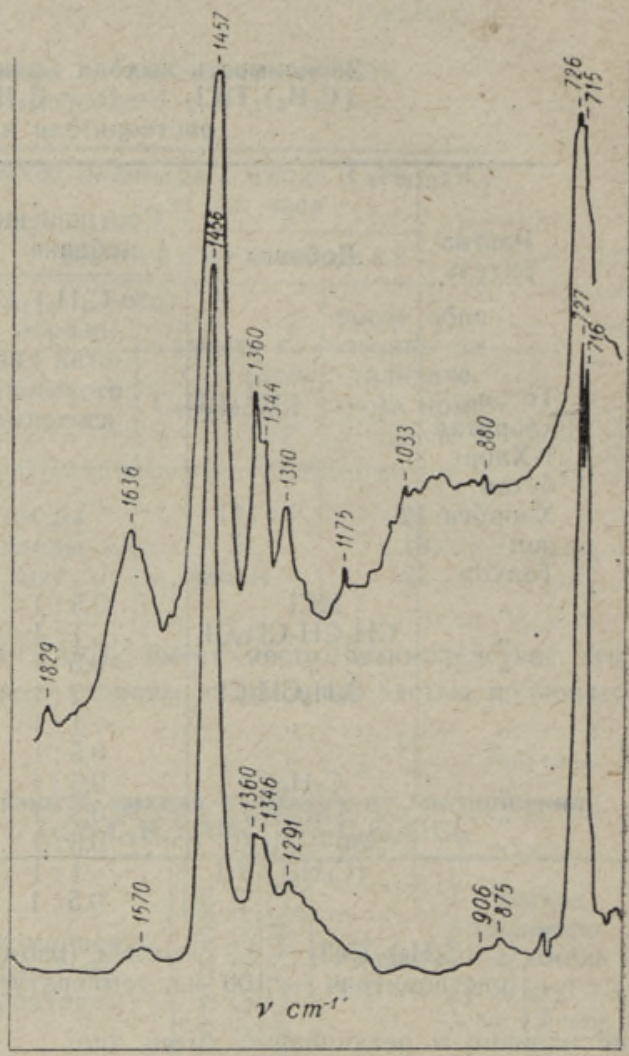

Рис. 4. Инфракрасный спектр полиэтилена, полученного на системе $\left(\mathrm{C}_{5} \mathrm{H}_{5}\right)_{2} \mathrm{TiCl}_{2}+$ $+\left(\text { uзo- } \mathrm{C}_{4} \mathrm{H}_{9}\right)_{2} \mathrm{AlCl}+\left(\mathrm{C}_{6} \mathrm{H}_{5}\right)_{3} \mathrm{CCl}$.

Внизу для сравнения приведен спектр линейного полиэтилена, полученного на системе $\mathrm{TiCl}_{4}+\left(\mathrm{C}_{2} \mathrm{H}_{5}\right)_{3} \mathrm{Al}$.

\section{и является менее кристаллическим}

(полоса $1310 \mathrm{~cm}^{-1}$ ). Температура плавления полимера более низкая $\left(128-129^{\circ}\right)$.

Это обусловлено тем, что рост полимерных цепей проходит на неодинаковых активных центрах и кординационная полимеризация осложняется чисто катионными процессами, связанными с наличием в системе стабильных карбкатионов.

\section{Выводы}

1. Каталитическая активность системы $\left(\mathrm{C}_{5} \mathrm{H}_{5}\right)_{2} \mathrm{TiCl}_{2}+\mathrm{R}_{2} \mathrm{AlCl}$ при полимеризации этилена повышается не только при добавленни небольших количеств $\mathrm{HCl}$ (что описано в литературе), но и при добавлении $\mathrm{RAlCl}_{2}$ или алкилгалогенидов, содержащих подвижный атом $\mathrm{Cl}$.

Действие добавок $\mathrm{HCl}$ и $\mathrm{R}^{1} \mathrm{Cl}$ связано с их взанмодействием с $\mathrm{R}_{2} \mathrm{AlCl}$, приводяция к образованию $\mathrm{RAICl}_{2}$.

2. Все указанные добавки повышают активность катализатора лишь в том случае, если их вводят в реакционную смесь после формирования каталитического комплекса, а не до или во время формирования.

3. Активирующее действие электрофильных добавок вызвано тем, что они взаимодействуют с анионной частью каталитического комплекса, разрыхляя полярную- связь между титаном и алюминием, вследствие этого облегчается внедрение по этой связи молекул мономера и уменьшается скорость внутрикомплексного диалкилирования титана. 


\title{
ЛИТЕРАТУРА
}

1. D. S. Breslow, N. R. New burg, J. Am. Chem. Soc., 1959, 81, 81.

2. D. S. B reslow, N. R. New burg, Chem. Eng. News, 1958, 36, 2956.

3. D. S. Breslow, N. R. New burg. J. Am. Chem. Soc., 1957, 79, 5072.

4. Hj. Sinn, H. Winter, W. v. T irpitz, Die Makromol. Chem., 1961, 48, 59.

5. A. Drucker, J. H. D a n iel, J. Polym. Sci., 1959, 37, 132, 553.

6. Х. Ка а р, О. Киррет, Г. Швиндлерман, Изв. АН ЭССР. Сер. физ.матем. н техн. наук, 1963, 3, 295.

7. А. К. Зе фирова, А. Е. Шилов, ДАН, 1961, 3, 599.

8. А. Е. Шил лов, А. К. Шил о в а, Б. Н. Боко в, Высокомолекулярные соедннення, $1962,11,1688$.

9. K. Z i egler, Organometallic Chemistry. Ed. by H. Zeiss, New York, 1960, 198.

10. Е. М юлле р, Новые воззрения в органнческой химин. ИЛ, 1960, 241.

\section{Ннститут хинии \\ Акадении наук Эстонской ССР \\ 20. VI 1963 \\ TITAANI TSUKLOPENTADIENÜULUUHENDITEL BASEERUVATE KATALOUTILISTE SÜSTEEMIDE AKTIIVSUSE UURIMINE ETULEENI POLOMERISATSIOONIL}

Поступнла в редакцию

\author{
H. Kaar \\ o. Kirret, \\ Eesti NSV Teaduste Akadeemia korrespondeeriv liige
}

G. Schwindlerman

Resümee

2. Katalüütilise kompleksi $\left(\mathrm{C}_{5} \mathrm{H}_{5}\right)_{2} \mathrm{TiCl}_{2}-\left(\text { iso- } \mathrm{C}_{4} \mathrm{H}_{9}\right)_{2} \mathrm{AlCl}$ aktiivsuse uurimine

Süsteemi $\left(\mathrm{C}_{5} \mathrm{H}_{5}\right)_{2} \mathrm{TiCl}_{2}-\left(\text { iso- } \mathrm{C}_{4} \mathrm{H}_{9}\right)_{2} \mathrm{AlCl}$ katalüütilist aktiivsust etüleeni polümerisatsioonil võib suurendada $\mathrm{HCl}$, liikuva klooriga alküülhalogeniidide või alküülalumiiniumdikloriidide lisamise abil.

Kōik ülalnimetatud lisandid tõstavad katalüsaatori aktiivsust sel puhul, kui neid viiakse süsteemi pärast katalüütilise kompleksi formeerumist.

Lisandite toime pōhineb nende mōjûl komp̉leksi anioonsele osale, mille tagajärjel kergeneb monomeeri molekulide sisestumine katalüütilise kompleksi katioonse ja anioonse osa vahele.

Eesti NSV Teaduste Akadeemia Keemia Instituut

Saabus toimetusse

20. VI 1963

\section{STUDY OF THE ACTIVITY OF CATALYTIC SYSTEMS \\ BASED ON BIS-CYCLOPENTADIENYL COMPOUNDS OF TITANIUM \\ IN POLYMERISATION OF ETHYLENE}

\author{
H. Kaar \\ O. Kirret, \\ Corresponding Member of the Academy of Sciences of the Estonian S. S. R.
}

G. Schwindlerman

Summary

II. Study of the activity of catalytic complex $\left(\mathrm{C}_{5} \mathrm{H}_{5}\right)_{2} \mathrm{TiCl}_{2}-\left(\text { iso- } \mathrm{C}_{4} \mathrm{H}_{9}\right)_{2} \mathrm{AlCl}$

The catalytic activity of the $\left(\mathrm{C}_{5} \mathrm{H}_{5}\right)_{2} \mathrm{TiCl}_{2}-\left(\text { iso- } \mathrm{C}_{4} \mathrm{H}_{9}\right)_{2} \mathrm{AlCl}$ system in the polymerisation of ethylene can be increased by adding $\mathrm{HCl}, \mathrm{RCl}$ or $\mathrm{RAlCl}_{2}$ containing a mobile atom of $\mathrm{Cl}$. An increase in the catalyst's activity occurs provided those substances are added after the formation of the catalytic complex. The effect of the substances added is due to their interaction with the anionic part of the complex, this interaction facilitating an inculcation of the Ti-Al bond by the molecules of the monomer.

Academy of Sciences of the Estonian S.S.R., Institute of Chemistry

Received June 20th, 1963 